\title{
NRO Legacy Project: Survey of Giant Molecular Clouds in M33
}

N. Kuno ${ }^{1,2}$, T. Tosaki ${ }^{3}$, S. Onodera ${ }^{1}$, K. Muraoka ${ }^{4}$, H. Kaneko ${ }^{2}$, T. Sawada ${ }^{5}$, K. Nakanishi ${ }^{2,5}$, S. Komugi ${ }^{6}$, Y. Tamura ${ }^{7}$, K. Kohno ${ }^{7}$, R. Kawabe ${ }^{1}$, N. Arimoto ${ }^{2,5}$ and S. Okamoto

${ }^{1}$ Nobeyama Radio Observatory, National Astronomical Observatory of Japan, ${ }^{2}$ Graduate

University for Advanced Studies (SOKENDAI), Japan, ${ }^{3}$ Joetsu University of Education, Japan, ${ }^{4}$ Osaka Prefecture University, Japan, ${ }^{5}$ National Astronomical Observatory of Japan, ${ }^{6}$ Insititute of Space and Astronautical Science, Japan Aerospace Exploration Agency, Japan,

${ }^{7}$ University of Tokyo, Japan

As the Nobeyama Radio Observatory Legacy Project: Survey of Giant Molecular Clouds in M33, we have been mapping M33 in $\mathrm{CO}(1-0)$ with the multi-beam receiver BEARS equipped on the 45-m telescope using the OTF mapping technique since 2007. The purpose of this project is to investigate the physical properties of GMCs and understand the evolutionary process from GMC formation to star formation in GMCs by comparing with various data such as $\mathrm{CO}(3-2), 1.1 \mathrm{~mm}$ continuum obtained with ASTE10m telescope at Atacama and the optical data obtained with SUBARU. We identified 87 GMCs using the first year data of $\mathrm{CO}(1-0)$ and observed 28 GMCs among them in $\mathrm{CO}(3-2)$ with ASTE (Onodera 2009, PhD thesis, University of Tokyo). From the comparison of these lines, it was shown that the $\mathrm{CO}(3-2) / \mathrm{CO}(1-0)$ ratio increases with star forming activity in the GMCs. Furthermore, we found that more massive GMCs tend to have higher $\mathrm{CO}(3-2) / \mathrm{CO}(1-0)$ ratio. Since the ratio is thought to be an indicator of the fraction of warm and dense molecular gas, our results imply that the fraction of warm and dense gas increases with GMC mass. Especially, since the ratio in the GMCs with low star forming activity is in the range where the ratio depends mainly on the density, we speculate that dense gas fraction increases with GMC mass.

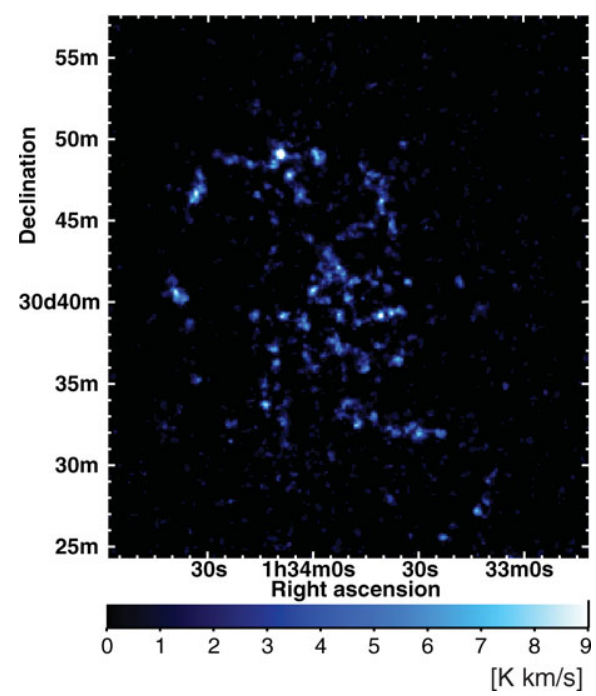

Figure 1. CO(1-0) integrated intensity map of M33. 